University of Nebraska - Lincoln

DigitalCommons@University of Nebraska - Lincoln

Faculty Publications from the Department of Electrical \& Computer Engineering, Department Electrical and Computer Engineering

5-1-1995

\title{
Determination of AIAs optical constants by variable angle spectroscopic ellipsometry and a multisample analysis
}

\author{
C. M. Herzinger \\ University of Nebraska-Lincoln
}

H. Yao

University of Nebraska-Lincoln

Paul G. Snyder

University of Nebraska-Lincoln, psnyder1@unl.edu

F. G. Celii

Texas Instruments, Central Research Lab., Dallas, Texas

Y. C. Kao

Texas Instruments, Central Research Lab., Dallas, Texas

See next page for additional authors

Follow this and additional works at: https://digitalcommons.unl.edu/electricalengineeringfacpub

Part of the Electrical and Computer Engineering Commons

Herzinger, C. M.; Yao, H.; Snyder, Paul G.; Celii, F. G.; Kao, Y. C.; Johs, B.; and Woollam, John A., "Determination of AIAs optical constants by variable angle spectroscopic ellipsometry and a multisample analysis" (1995). Faculty Publications from the Department of Electrical and Computer Engineering. 58. https://digitalcommons.unl.edu/electricalengineeringfacpub/58

This Article is brought to you for free and open access by the Electrical \& Computer Engineering, Department of at DigitalCommons@University of Nebraska - Lincoln. It has been accepted for inclusion in Faculty Publications from the Department of Electrical and Computer Engineering by an authorized administrator of DigitalCommons@University of Nebraska - Lincoln. 


\section{Authors}

C. M. Herzinger, H. Yao, Paul G. Snyder, F. G. Celii, Y. C. Kao, B. Johs, and John A. Woollam 


\title{
Determination of AlAs optical constants by variable angle spectroscopic ellipsometry and a multisample analysis
}

\author{
C. M. Herzinger, H. Yao, and P. G. Snyder \\ Center for Microelectronic and Optical Materials Research, and Department of Electrical Engineering, \\ University of Nebraska-Lincoln, Lincoln, Nebraska 68588-0111
}

F. G. Celii and Y.-C. Kao

Texas Instruments, Central Research Lab., Dallas, Texas 75265

B. Johs and J. A. Woollam

J. A. Woollam Co., Lincoln. Nebraska 68588-0111

(Received 25 August 1994; accepted for publication 11 January 1995)

\begin{abstract}
Using variable angle spectroscopic ellipsometry, optical constants for AlAs (1.4-5.0 eV) are presented which are simultaneously compatible with measured data from four different samples. The below-gap index values are compatible with published prism measured values. The second derivative spectrum are compatible with published values above the direct band gap. The AlAs spectra is Kramers-Kronig self-consistent over the measured range and is compatible with published values from 0.6 to $1.4 \mathrm{eV}$. The optical constants for thin $(<50 \AA) \mathrm{GaAs}$ caps on AlAs are shown to be different from bulk GaAs values and require special consideration when fitting ellipsometric data. For the thin GaAs caps, the $E_{1}$ and $E_{1}+\Delta_{1}$ critical-point structure is shifted to higher energies as previously observed for GaAs quantum wells. Bulk AlAs optical constants are shown to be different from those of a thin $(\sim 20 \AA)$ AlAs barrier layer embedded in GaAs. The thin barrier layer exhibits a highly broadened critical-point structure. This barrier broadening effect (AlAs) and the thin cap shifting effects (GaAs) have implications for in situ growth control schemes which make use of the $E_{1}$ and $E_{1}+\Delta_{1}$ critical-point region. (C) 1995 American Institute of Physics.
\end{abstract}

\section{INTRODUCTION}

Nondestructive optical techniques, such as variable angle spectroscopic ellipsometry (VASE), are attractive for semiconductor heterostructure characterization, provided accurate dielectric functions are available for the constituent layers. AlAs is one end point of the heavily studied $\mathrm{Al}_{x} \mathrm{Ga}_{1-x}$ As ternary system; however, its optical properties have been difficult to measure due to its extreme reactivity with oxygen and water vapor. For this work, VASE was used to determine thicknesses and optical constants for AlAs epitaxial layers. This was done by employing a powerful multisample data analysis technique. Direct VASE measurement of bulk AlAs optical constants using chemically cleaned and polished samples is impossible due to rapid surface oxidation. ${ }^{1}$ For this work, samples were measured both in situ, inside an ultrahigh-vacuum (UHV) chamber, and ex situ, on the bench top. The in situ sample was transported to the measurement site with an amorphous As (a-As) passivation layer which was later desurbed at $\sim 400^{\circ} \mathrm{C}$ in the UHV chamber. The ex situ samples were capped with $\mathrm{GaAs}$ to protect the more reactive AlAs layers.

Except for the case of a bare substrate, determining optical constants from VASE data requires the fitting of a parameterized model to the measured data. Even a native oxide layer which is optically thin and has low dispersion is a complication when trying to determine the optical constants of the underlying material. ${ }^{2-4}$ For the ex situ samples, semiconductor (GaAs) caps were used to protect the AlAs layers. With both a semiconductor and an oxide overlayer present, the data modeling becomes very complicated and the underlying optical constants are extremely difficult to obtain if only one sample is available..$^{5}$ An $a$-As decapped sample can provide more direct information about the AlAs, but the desorption process produces a roughness/residual-As layer of indeterminate composition and thickness. ${ }^{6,7}$ For both in situ and ex situ samples, the overlayer structure must be determined in order to extract the AlAs optical constants. VASE measurements were performed; however, data acquisition at multiple angles is insufficient to uniquely determine the nature of an optically thin overlayer ${ }^{4}$ on a single sample. For example, extrapolation of the expected $\epsilon_{2}$ peak height at the $E_{2}$ energy from published alloy values ${ }^{1}$ has been used as one possible criterion. ${ }^{5}$ In this work, we apply a powerful multisample data analysis technique which simultaneously analyzes data from samples with different layer thicknesses, but assumes the optical constants are the same for each sample. The concept of multisample analysis for ellipsometry has existed for some time as simulation studies ${ }^{8,9}$ and has recently been used as a primary tool for investigating actual thin-film samples. ${ }^{10,11}$

We present results of multisample analyses utilizing measurements of three GaAs-capped AlAs samples (ex situ) and one As-decapped sample in a UHV chamber (in situ). By simultaneously fitting the data from all samples, we have determined both bulk AlAs optical constants and layer thicknesses for the four samples. A sequence of fitting procedures is presented to illustrate the model refinements needed to fully explain the measured data. As a key refinement, we demonstrate that the optical constants of the thin GaAs cap layers on the ex situ samples require special consideration to obtain satisfactory model fits. We compare the extracted AlAs optical constants with previously published values. ${ }^{6,12}$ 
We also demonstrate the utility of the new AlAs values in determining layer thicknesses for individual samples grown at different temperatures. Last, we show that the optical constants for an isolated, thin $(\sim 20 \AA)$ AlAs barrier in GaAs is poorly described by bulk AlAs values.

\section{EXPERIMENT AND VASE BASICS}

Three ex situ samples (numbered 7179, 7357, and 7358) with different AlAs layer thicknesses (nominally 1000, 800, and $700 \AA$ ) were grown on GaAs substrates at $600^{\circ} \mathrm{C}$ and capped with nominally $50 \AA$ of GaAs. A fourth sample $(7180,1000 \AA)$ was grown at $450^{\circ} \mathrm{C}$ for comparison. The in situ AlAs layer $(4500 \AA)$ was grown at $600^{\circ} \mathrm{C}$ and capped in an As flux after the substrate was cooled to below room temperature. The decapping and measurement procedures for the in situ sample are described more fully in Ref. 7 . The standard ellipsometric parameters $\psi$ and $\Delta$ were measured on each ex situ sample for photon energies from 1.42 to $5.0 \mathrm{eV}$ $\left(0.01 \mathrm{eV}\right.$ steps) at incident angles of $72.5^{\circ}$ and $75^{\circ}$ using a $\mathrm{J}$. A. Woollam Co., variable angle scanning rotating-analyzer ellipsometer. The in situ sample was measured over the same spectral range at an incident angle of $74.1^{\circ}$. However, because the in situ sample had a much thicker AlAs layer and was measured prior to a monochromator improvement, the interference fringes were not measured as accurately as for the ex situ samples. Therefore only in situ data from the AlAs absorbing region above the direct band gap (3.2-5.0 eV) were included in the fitting process. Because only one incident angle was available on the UHV chamber, the in situ data were included twice to keep the fit weighting equal relative to the ex situ samples which were measured at two incident angles.

Ellipsometry determines thicknesses and optical constants for layered samples by fitting the measured data to a parameterized model. The standard model for analyzing VASE data is a sequence of parallel layers with smooth interfaces and homogenous optical constants, on a semiinfinite substrate. ${ }^{13}$ Our fitting procedure is described more fully elsewhere, ${ }^{4}$ but the basic terminology is given below. The standard ellipsometric parameters $\psi$ and $\Delta$ are related to the complex ratio of reflection coefficients for light polarized parallel $(p)$ and perpendicular $(s)$ to the plane of incidence. ${ }^{13}$ This ratio is defined as

$$
\rho=\frac{R_{p}}{R_{s}}=\tan (\psi) e^{i \Delta}
$$

The electric-field reflection coefficient for $p(s)$-polarized light is given by $R_{p}\left(R_{s}\right)$. In addition to $\psi$ and $\Delta$, their standard deviations, $\sigma_{\psi}^{\exp }$ and $\sigma_{\Delta}^{\exp }$, are measured using multiple revolutions of the analyzer. (Multiple revolutions are used in any case to improve the signal-to-noise ratio for $\psi$ and $\Delta$.) The Levenberg-Marquardt algorithm ${ }^{14}$ is used to fit the model parameters by minimizing the following weighted (biased) test function: ${ }^{15}$

$$
\begin{aligned}
\xi^{2} & =\frac{1}{2 N-M} \sum_{i=1}^{N}\left[\left(\frac{\psi_{i}^{\bmod }-\psi_{i}^{\text {exp }}}{\sigma_{\psi, i}^{\exp }}\right)^{2}+\left(\frac{\Delta_{i}^{\bmod }-\Delta_{i}^{\text {exp }}}{\sigma_{\Delta, i}^{\exp }}\right)^{2}\right] \\
& =\frac{1}{2 N-M} \chi^{2} .
\end{aligned}
$$

The number of measured $\psi$ and $\Delta$ pairs is $N$ and the total number of real valued fit parameters is $M$. The figure of merit (FOM) we use to describe confidence in the $i$ th fit parameter is given by

$$
\mathrm{FOM}_{i}=1.65 \xi \sqrt{C_{i i}} \text {. }
$$

This is the usual one-parameter, $90 \%$, uncorrelated confidence limit ${ }^{14}$ multiplied by our test function $\xi$, where $C_{i i}$ is the $i$ th diagonal element of the fit parameter covariance matrix. ${ }^{4}$ In the case of a good fit with no systematic errors, $\xi^{2}$ tends toward a value of one and $\mathrm{FOM}_{i}$ reduces to the standard $90 \%$ confidence limit. This FOM combines information about the sharpness of the fit minimum $\left(C_{i l}\right)$ with information about the overall quality of the fit. The FOM is primarily related to the combined measurement and fitting process. Using the FOM as direct quantitative information about the sample is only valid when $\sigma_{\psi}^{\exp }$ and $\sigma_{\Delta}^{\exp }$ are known to be accurate in magnitude, and when random (not systematic) measurement errors dominate the fit. ${ }^{4}$

\section{TABULATED AND PARAMETRIC OPTICAL CONSTANTS}

Tabulated optical constants are defined by a wavelengthby-wavelength list of the dielectric function. Parametric optical constants, in contrast, use a physically or empirically based mathematical model. Both tabulated and parametric optical constants are used in this work.

Tabulated optical constant lists can be the result of fitting spectroscopic ellipsometry (SE) data or they might be taken from previous publications utilizing other characterization methods. When analyzing SE data, tabulated optical constants are convenient to work with as fit parameters because the complex dielectric values at different wavelengths do not depend on each other. 'The general difficulty with tabulated optical constants, however, is that the number of model unknowns is directly proportional to the number of measurement wavelengths. On the other hand, for a given spectral range, parametric optical constants have a fixed number of defining parameters which is independent of the measured wavelength density. Thus, by increasing the density of measured wavelengths, one can increase the number of independent measurements without increasing the number of model unknowns.

Parametric optical constants models are possible because real materials do not have random diclectric functions and there is a physical relationship, the Kramers-Kronig (K-K) integral, between the real and imaginary parts, $\epsilon_{1}(\hbar \omega)$ and $\epsilon_{2}(\hbar \omega)$. Furthermore, dielectric functions are superpositional and can be built up from pieces that are each $\mathrm{K}-\mathrm{K}$ consistent. Parametric models allow the determination of optical constants over a wide spectral range by fitting only a few parameters. Also; these models prevent wavelength-to- 
wavelength measurement noise from becoming part of the extracted optical constants which would be the case with tabulated optical constants. This smoothing feature exists because the possible range of dielectric functions is limited by the model definition. The same property that smoothes out noisy data, however, can also smooth away or distort real spectral features. Or worse; the model may not possess enough flexibility and large systematic errors will exist between the model and the data in certain spectral regions. When available, parametric models can greatly reduce the number of fit parameters, but they require attention to ensure sufficient flexibility.

For ellipsometric data analysis, the Cauchy model for dielectrics ${ }^{16}$ and the Zollner model for semiconductor oxides $^{17}$ are examples of useful parametric models. However, no general parametric model has yet been developed that can completely describe a semiconductor's critical-point (CP) structure and also be used to extract optical constants from raw VASE data of layered samples. One complex model has been developed to describe a semiconductor dielectric function and its derivatives. ${ }^{18}$ This model can be fit to previously measured dielectric functions revealing information about $\mathrm{CP}$ energies, $\mathrm{CP}$ broadenings, and the joint density of states. However, the two stage fitting process employed is not applicable when directly fitting. VASE data to obtain the dielectric function. A less ambitious model, an oscillator ensemble, has been used to model semiconductor CP structures for energies above the direct band gap. ${ }^{19-22}$ This modeling technique employs oscillators at the major critical points, with extra "fictitious" oscillators to fill in the absorption between critical points. Oscillators cannot describe the sharp change in absorption at the lowest energy direct gaps, however, and an oscillator ensemble is usually a very poor model below and just above such. a CP. In this work we employed an oscillator model [Eq. (4)] as an intermediate step in determining layer thicknesses when analyzing the dielectric function of the three thin GaAs cap layers:

$$
\epsilon(\hbar \omega)=\epsilon_{1}^{\mathrm{offset}}+\sum_{j=1}^{N} \frac{A_{j}}{E_{j}^{2}-(\hbar \omega)^{2}-i B_{j} \hbar \dot{\omega}} .
$$

The direct gap of $\mathrm{GaAs}$ at $1.42 \mathrm{eV}$ was not a problem since it is outside the primary spectral range of interest for AlAs. An oscillator model as in Eq. (4) has been used for AlAs above the gap and at high temperatures. ${ }^{7}$ However, for this work we are interested in a spectral region including the lowest direct band gap at $3 \mathrm{eV}$. Therefore tabulated optical constants are used for determining the AlAs dielectric function.

\section{DATA ANALYSIS AND DISCUSSION}

The usual method for determining optical constants from single-sample VASE data is to set up the fitting model with fixed layer thicknesses, use previously determined optical constants for all layers not of interest, and then fit optical constants at each wavelength for the one layer of interest. If only single angle SE data is available, this list represents the maximum information that can be extracted from the mea- sured spectrum because the number of data values and fit parameters is equal. Because SE is very sensitive, precise knowledge of layer thicknesses is required to obtain accurate optical constants.

Use of parametric optical constants would reduce the number of unknowns and potentially allow more fit parameters while still maintaining an overdetermined model (more measurements than fit parameters). However, parametric optical constant models do not remove the correlation between dielectric function amplitude and layer thicknesses when fitting. Therefore single angle SE data is insufficient to independently determine the dielectric function and the thickness of a layer.

Acquiring data at multiple angles is a useful technique to increase the number of measured data points without increasing the number of measurement wavelengths. In theory, controllably changing the path length of the probe beam inside the sample by varying the angle of incidence increases the number of model parameters that can be independently determined. However, when a single sample is measured at multiple angles and the model is overdetermined, unique optical constants and layer thicknesses still cannot generally be determined due to correlation between these model parameters. This is especially true for high index materials like semiconductors wherc the probe beam is strongly refracted and travels nearly the same path inside the sample for a wide range of incident angles. The primary use of multiple incidence angles on semiconductors is to ensure that for all spectral regions some data is acquired near the optimal measurement regime of the type of ellipsometer being used. ${ }^{4}$ For a rotating-analyzer ellipsometer, this occurs for $\Delta \sim 90^{\circ}{ }^{23}$

Intensity transmission data, if available, can greatly reduce the correlation between dielectric function and thickness. ${ }^{24}$ For this work, however, AlAs was grown on GäAs substrates, and GaAs has no transparent spectral region through which to measure the AlAs absorption strength as $E_{g}^{\mathrm{GaAs}}<E_{g}^{\mathrm{AlAs}}$. In principle, the GaAs substrate could be polished thin enough to allow a transmission measurement, but this would have been extremely difficult and was unnecessary because multiple samples were available.

For this work, we employed a multisample analysis technique in which VASE data from four samples were fit simultaneously to four models with different thicknesses but the

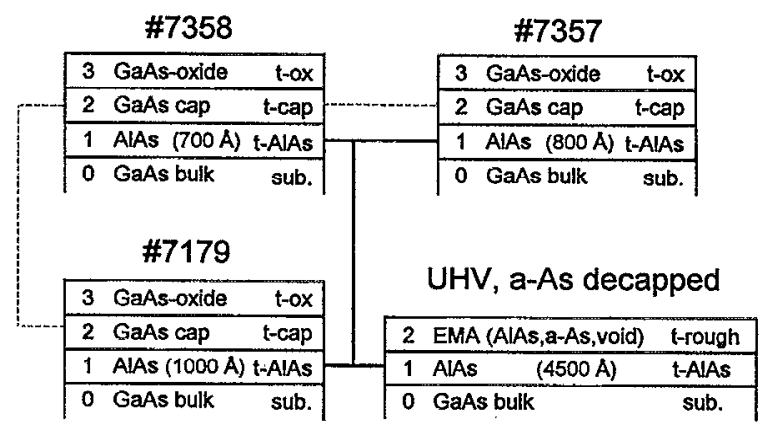

FIG. 1. Models used to simultaneously fit the measured ellipsometric data. The solid lines indicate that the AlAs optical constants were the same for all four models. The dashed lines indicate that same GaAs cap optical constants were used for the three ex situ samples. 
TABLE I. Summarized fit procedures for ex situ GaAs cap optical constants, AlAs optical constants, and structural parameters.

\begin{tabular}{|c|c|c|c|}
\hline Fit & GaAs cap layers & AlAs & Thicknessess \\
\hline 0 & $\begin{array}{c}\text { tabulated bulk (Ref. } 25 \text { ) } \\
\text { not fit }\end{array}$ & $\begin{array}{c}\text { previously } \\
\text { published (Ref. 6) }\end{array}$ & fit all \\
\hline 1 & $\begin{array}{c}\text { tabulated bulk (Ref. 25) } \\
\text { not fit }\end{array}$ & fit & fit all \\
\hline 2 & $\begin{array}{l}\text { oscillators bulk } \\
\text { not fit }\end{array}$ & fit & fit all \\
\hline 3 & $\begin{array}{l}\text { oscillators } \\
\text { fit } E_{1} \text { and } E_{1}+\Delta_{1}\end{array}$ & fit & fit all \\
\hline 4 & $\begin{array}{l}\text { tabulated } \\
\text { fit }\end{array}$ & fit & $\begin{array}{c}t \text {-ox } / t \text {-cap from } 3 \\
\text { fit others }\end{array}$ \\
\hline 5 & $\begin{array}{l}\text { tabulated } \\
\text { fit }\end{array}$ & $\begin{array}{c}1.42-5.0 \text { by } 0.01 \mathrm{eV} \\
\text { fit }\end{array}$ & $\begin{array}{c}\text { from fit } 4 \\
\text { not fit }\end{array}$ \\
\hline 6 & $\begin{array}{l}\text { tabulated } \\
\text { fit }\end{array}$ & fit & fit all \\
\hline 7 & $\begin{array}{c}\text { tabulated } \\
\text { fit }\end{array}$ & $\begin{array}{c}\text { previously } \\
\text { published (Ref. 6) }\end{array}$ & fit all \\
\hline 8 & $\begin{array}{c}\text { tabulated bulk (Ref. 25) } \\
\text { not fit }\end{array}$ & $\begin{array}{c}\text { from fit } 5 \\
\text { not fit }\end{array}$ & fit all \\
\hline
\end{tabular}

same optical constants. Figure 1 shows the layered models used for the four samples. Tabulated optical constants for bulk material were used for the substrate $\mathrm{GaAs},{ }^{25}$ the amorphous As (a-As), ${ }^{26}$ and the GaAs oxide. ${ }^{17}$ For the fitting process, 13 structural parameters were used which consisted of 11 thicknesses (ex situ: 3 samples $\times 3$ layers; in situ: 2 layers) and two volume fractions (in situ). The three constituent Bruggeman effective-medium approximation (EMA) layer $^{27}$ was included in the in situ sample model because the true microstructure of the surface was unknown, and this type of layer allowed a wide range of overlayer descriptions. For this EMA layer, individual volume fractions are not very meaningtul because there is correlation among them and with the layer thickness. The lines in Fig. 1 indicate coupling of optical constants between layers of different models. The $\mathrm{GaAs}$ substrate and GaAs-oxide optical constants were never allowed to vary as fit parameters and were, therefore, not explicitly coupled. The optical constants of the AlAs and the thin $\mathrm{GaAs}$ cap layers. were allowed to be adjustable for the fit as a whole, but not for individual samples. We show through the sequence of fitting procedures presented that the thin GaAs cap optical constants required special consideration because bulk GaAs values were inadequate. For the majority of the fits described, only a subset of the acquired data (1.8 $5.0 \mathrm{eV}$ in $0.02 \mathrm{eV}$ steps) was used. The step size increase was required to keep the number of fit parameters reasonable while working with tabulated AlAs optical constants. The reduction in spectral range was required to use the parametric model (Sec. III) for the GaAs cap layers. For all fit procedures that had adjustable thickness parameters, this same reduced data set was used. With the thicknesses fixed, the final optical constants were extracted on a wavelength-bywavelength basis from the full data set. The fitting procedures and resulting structural parameter fit values are summarized in Tables I and II, respectively.

As a base line, fit 0 used published bulk $\mathrm{AlAs}^{6}$ and bulk cap $\mathrm{GaAs}^{25}$ optical constants to fit the 13 structural parameters. No optical constants were fit and as such this first fit was not a true use of the multisample technique. The final results for both the averaged $\chi^{2}$ and for our test function $\xi^{2}$ are included in Table II, as is the FOM for each parameter as defined in Eq. (3). The averaged $\chi^{2}$ is just a weighted measure of the closeness between the data and model, whereas $\xi^{2}$ is more directly. related to the statistics of the fit and depends on the number of parameters being fit, $M$. The FOM is best used on a relative basis when comparing parameters

TABLE II. Summary of structural parameter fit results. Thicknesses in angstroms, compositions in \%, FOM [Eq. (3)] in parentheses.

\begin{tabular}{|c|c|c|c|c|c|c|c|c|c|}
\hline & Fit & 0 & 1 & 2 & 3 & 4 & 6 & 7 & 8 \\
\hline & $M$ & 13 & 335 & 335 & 341 & 651 & 657 & 335 & 13 \\
\hline & $\xi^{2}$ & 2087 & 383 & 393 & 51.5 & 15.1 & 13.5 & 363 & 874 \\
\hline Sample & $\frac{1}{2 N} \chi^{2}$ & 2072 & 308 & 316 & 41.3 & 9.7 & 8.6 & 292 & 868 \\
\hline \multirow{3}{*}{7358} & $t$-ox & $31.5(0.29)$ & $30.9(0.11)$ & $31.2(0.11)$ & $32.5(0.06)$ & 32.5 & $35: 1(0.02)$ & $11.5(0.12)$ & $31.4(0.19)$ \\
\hline & $t$-cap & $43.3(0.36)$ & $41.7(0.14)$ & $41.6(0.14)$ & $42.9(0.18)$ & 42.9 & $47.2(0.03)$ & $24.4(0.08)$ & $42.6(0.24)$ \\
\hline & $t$-AlAs & $729.9(0.75)$ & $714.9(0.29)$ & $715.5(0.29)$ & $709.3(0.22)$ & $709.8(0.05)$ & $704.1(0.05)$ & $765.1(0.28)$ & $717.7(0.48)$ \\
\hline \multirow{3}{*}{7357} & $t-\mathrm{Dx}$ & $32.6(0.44)$ & $32.8(0.17)$ & $33.1(0.17)$ & $34.6(0.07)$ & 34.6 & $37.0(0.03)$ & $14.4(0.20)$ & $33.0(0.28)$ \\
\hline & $t$-cap & $40.4(0.61)$ & $39.0(0.25)$ & $39.0(0.25)$ & $40.8(0.19)$ & 40.8 & $45.2(0.05)$ & $22.9(0.13)$ & $40.9(0.38)$ \\
\hline & $t$-AlAs & $831.6(1.21)$ & $819.1(0.48)$ & $819.6(0.49)$ & $819.6(0.24)$ & $819.9(0.08)$ & $814.1(0.08)$ & $873.8(0.45)$ & $820.9(0.77)$ \\
\hline \multirow{3}{*}{7179} & $t$-ox & $29.7(0.43)$ & $29.4(0.17)$ & $29.6(0.17)$ & $32.4(0.08)$ & 32.4 & $34.5(0.03)$ & $8.1(0.17)$ & $29.9(0.28)$ \\
\hline & $t$-cap & $52.7(0.50)$ & $51.3(0.19)$ & $51.4(0.19)$ & $47.1(0.20)$ & 47.1 & $53.2(0.03)$ & $27.7(0.10)$ & $50.9(0.32)$ \\
\hline & $t$-AlAs & $1008.7(1.13)$ & $992.4(0.44)$ & $993.4(0.44)$ & $999.5(0.23)$ & $1000.3(0.06)$ & $992.3(0.08)$ & $1058.1(0.36)$ & $994.5(0.74)$ \\
\hline \multirow{4}{*}{ in situ } & $\%$ a-As & $50.4(44.9)$ & $29.7(2.5)$ & $31.4(2.5)$ & $47.0(6.2)$ & $41.6(1.4)$ & $41.7(01.3)$ & $28.5(8.4)$ & $41.7(13.2)$ \\
\hline & $\%$ void & $18.8(12.6)$ & $18.8(2.4)$ & $18.8(2.4)$ & $23.3(2.1)$ & $20.5(0.7)$ & $20.5(0.6)$ & $18.8(4.7)$ & $20.5(6.4)$ \\
\hline & $t$-rough & $28.5(22.3)$ & $38.9(3.9)$ & $37.0(3.9)$ & $30.1(3.3)$ & $32.5(1.1)$ & $33.9(1.0)$ & $50.4(16.8)$ & $32.9(9.9)$ \\
\hline & $t$-AlAs & $4350.2(71.5)$ & $4287.1(29.2)$ & $4293.7(29.1)$ & $4335.6(7.0)$ & $4338.7(3.3)$ & $4339.8(3.1)$ & $4350.1(26.8)$ & $4339.9(31.4)$ \\
\hline
\end{tabular}



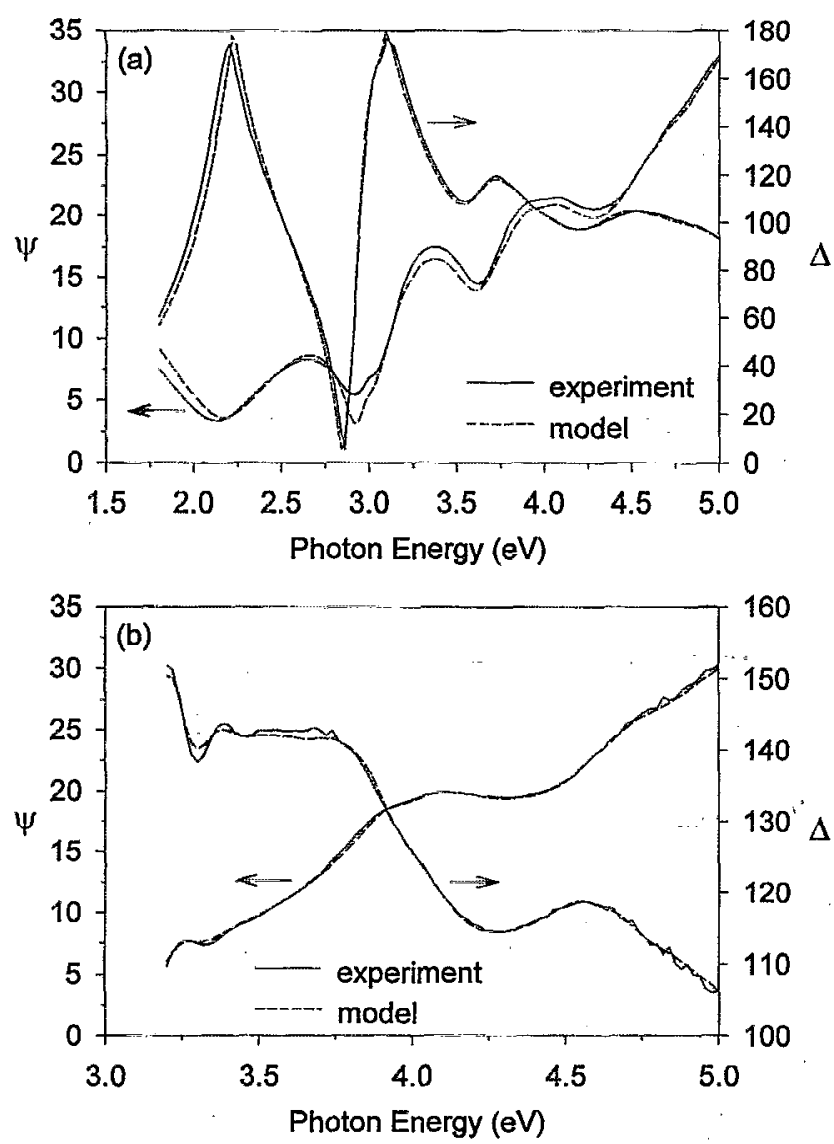

FIG. 2. The experimental data and model fits (a) for ex situ samples 7357 and (b) for the in situ sample are shown for fit 0 . The model used published AlAs optical constants and bulk GaAs optical constants for thie caps. Of the four samples, the in situ data is modeled the best. The ex situ data fits are poor and qualitatively similar to (a) for samples 7179 and 7357 . They do not match the interference pattern below $3 \mathrm{eV}$ and have substantial errors up to $4.5 \mathrm{eV}$.

from the same sample or for the same parameter in different fits. As demonstrated by the large $\chi^{2}$ and as shown in Fig. 2, the fits for the ex situ samples are inadequate [Fig. 2(a)] while the in situ data are fit quite well [Fig. 2(b)]. Our in situ data are at least compatible in shape with the ultraviolet (UV) published optical constants, ${ }^{6}$ but for the ex situ data there are definitely some problems with the optical constants or with the models.

The first true use of the multisample technique was fit 1 . For this fit, bulk GaAs was used for the cap and the AlAs dielectric values were fit at each wavelength simultaneously with the structural parameters. A total of 335 parameters were fit and the $\chi^{2}$ was reduced an order of magnitude (Table II). For this fit and for all succeeding optical constant fits, the imaginary part of the dielectric function was not allowed to go negative in the fitting algorithm. Figure 3 shows the resulting fits for ex situ sample 7357 and the in situ sample. Note especially that the interference oscillations below $3 \mathrm{eV}$ now match more closely. The multisample technique is most sensitive when the AlAs is transparent because the data for the three different sample thicknesses had to be fit with only one dielectric value at each wavelength. This is similar to the
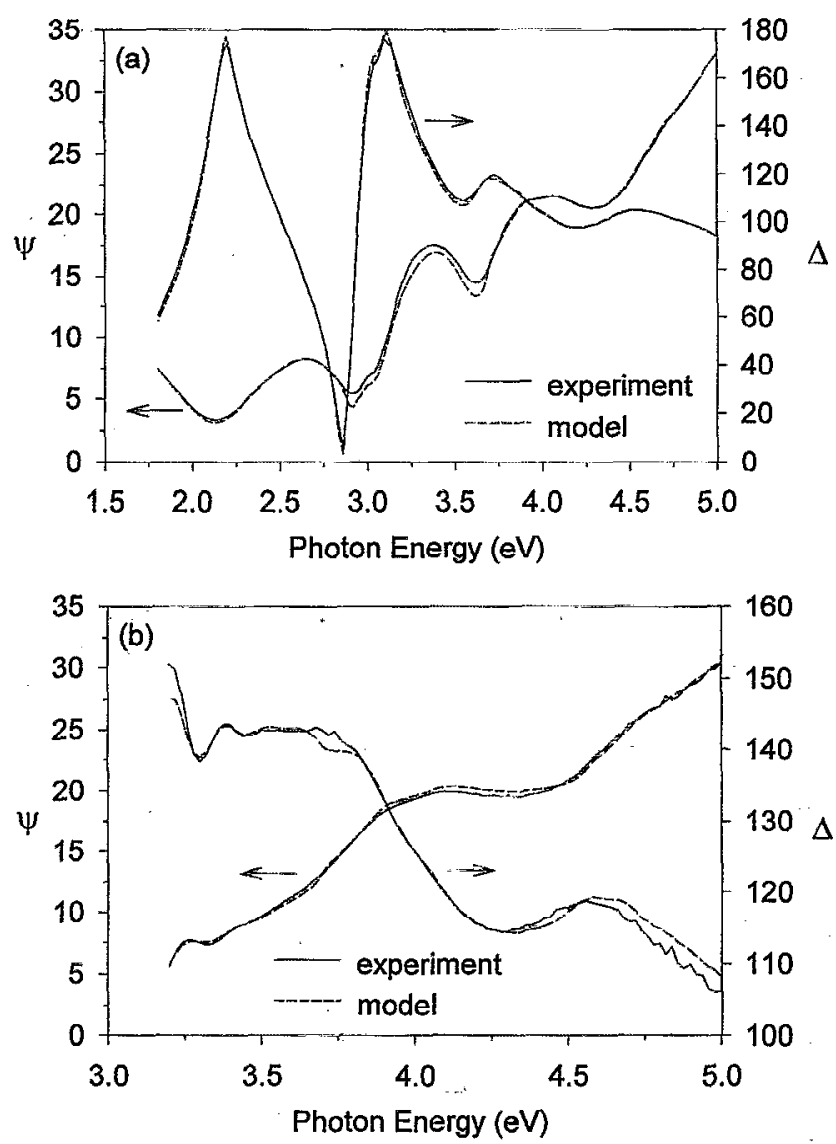

FIG. 3. The experimental data and model fits (a) for ex situ sample 7357 ( $800 \AA$ ) and (b) for the in situ sample are shown for fit 1 . The model used bulk GaAs optical constants for the caps and fit the AlAs optical constants. The other ex situ data fits are similar to (a).

idea of using multiple angles of incidence which attempts to controllably change the probe beam path length inside the material of interest. However, changing the path length by changing samples is much more effective than changing angles of incidence on a single sample.

There are still some problems in the region around $3 \mathrm{eV}$ which encompasses the AlAs $E_{0}$ and GaAs $E_{1}$ and $E_{1}+\Delta_{1}$ critical points. The in situ fit is not as good as for fit 0 [Fig. 3(b)], but this is not unexpected since the multisample analysis is globally trying to find a set of AlAs values that fits all the samples. Thus the optical constant coupling causes each sample model to have similar error levels. The AlAs dielectric function from fit 1 is compared with published values in Fig. 4. The comparison shows general shape agreement, but lesser agreement in $\epsilon_{2}$ peak heights which is related to overlayer modeling. The fit $1 \mathrm{AlAs}$ values have a spurious feature at $3.7 \mathrm{eV}$ which appears strongly in the derivative spectra (not shown) and is not present in the published values. This feature along with the imperfect fit indicate that the model is still inadequate. A closer look at the below-gap AlAs optical constants is made in Fig. 5, where the results of fit 1 are compared with published prism measurements. ${ }^{12}$ The angle of minimum deviation through a prism method is an accurate technique for measuring the refractive index of transparent material. ${ }^{28}$ The fit $1 \mathrm{AlAs}$ values are much closer to the prism 


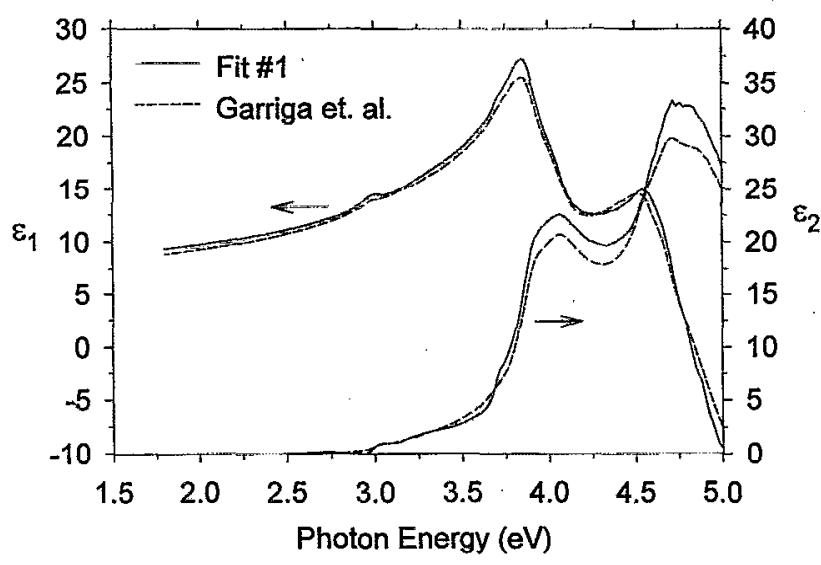

FIG. 4. The AlAs optical constants from fit 1 are compared with previously published values also determined by spectroscopic ellipsometry (Ret. 6). 'The offset in $\epsilon_{1}$ below $3 \mathrm{eV}$ explains the improved interference pattern fitting when comparing Fig. 3 to Fig. 2. The difference in $\epsilon_{2}$ above $3.8 \mathrm{eV}$ is related to the different cap layers assumed in this analysis and the published values. The anomalous feature at $3.7 \mathrm{eV}$ in the fit 1 spectra is unphysical.

measured values than are the previous ellipsometric measurements. Looking below $3.0 \mathrm{eV}$ in Fig. 4, the values do not appear very different, but the multisample technique is very sensitive in this region and produces good agreement with the prism values for fits $1-4$. The discrepancy with the previous Garriga SE measurement is primarily a result of the different experimental goals; the Garriga results were principally a study of $\mathrm{CP}$ energies as a function of temperature, and the below-gap refractive index was not an essential factor for that work. Our goal was to find AlAs optical constants that can be generally used for layer thickness determination. As such we were very interested in accurate below-gap values and more tolerant of noise in the UV region as seen in our in situ data. The fits shown in Fig. 3(a) are still not perfect especially from about 2.8 to $3.8 \mathrm{eV}$. The oxide optical constants are quite featureless, and any small offset in refractive index would be correlated with the oxide layer thicknesses and would have already been accounted for in the

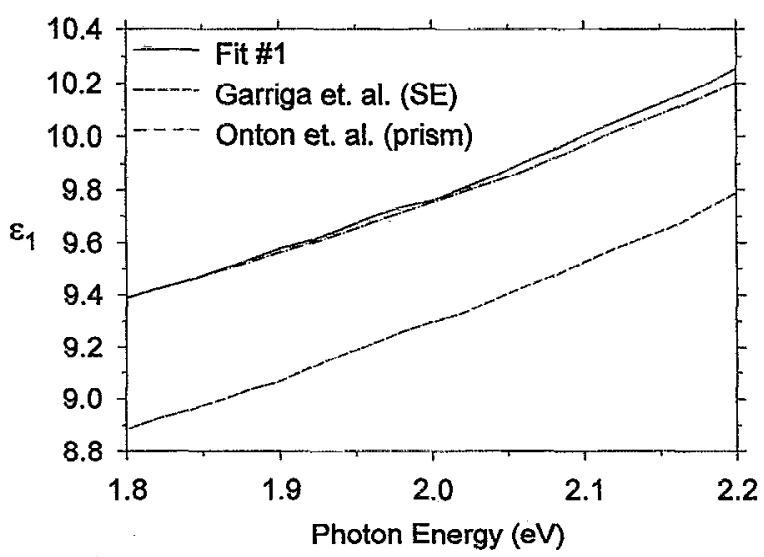

FIG. 5. Comparison of fit $1 \epsilon_{1}$ values with previously published ellipsometry (Ref. 6) and prism (Ref. 12) determined values. The fit 1 values closely agree with the prism measurements. The multisample measurement/analysis technique is a powerful technique for determining both optical constants and thicknesses when interference oscillations are present.
TABLE III. Oscillator cap fit parameters for fits 2 and 3.

\begin{tabular}{cccc}
\hline \multirow{2}{*}{ Critical point } & $\begin{array}{c}\text { Oscillator } \\
\text { parameter }\end{array}$ & $\begin{array}{c}\text { Bulk GaAs } \\
\text { fit 2 }\end{array}$ & $\begin{array}{c}\text { Cap GaAs } \\
\text { fit 3 }\end{array}$ \\
\hline \multirow{3}{*}{$E_{1}$} & $E(\mathrm{eV})$ & 2.922 & 2.977 \\
& $B(\mathrm{eV})$ & 0.171 & 0.239 \\
& $A\left(\mathrm{eV}^{2}\right)$ & 4.30 & 3.59 \\
$E_{1}+\Delta_{1}$ & $E(\mathrm{eV})$ & 3.136 & 3.167 \\
& $B(\mathrm{eV})$ & 0.377 & 0.541 \\
& $A(\mathrm{eV})$ & 12.98 & 18.32 \\
\hline \hline
\end{tabular}

model. The absorption strength of the AlAs layers above 3 $\mathrm{eV}$ is too great for the GaAs substrate optical constants to have much influence. The assumption of bulk optical constants for the thin GaAs cap layers, however, is possibly the problem.

Various model nonidealities including interfaces and $\mathrm{AI}$ presence in the cap layers $\left(\mathrm{Al}_{x} \mathrm{Ga}_{1-x} \mathrm{As}\right.$ caps) were considered in conjunction with these first two fits. For the lower GaAs/AlAs interface, an additional layer of $\mathrm{Al}_{0.5} \mathrm{Ga}_{0.5} \mathrm{As}$ or an EMA layer mixing equal parts GaAs and AlAs were considered, but there is little sensitivity to the detail of this interface, and the fits did not significantly improve. Furthermore, the thickness of this lower interface did not fit to a consistent value for the ex situ samples, indicating that the interfacial grading (if any) is below the sensitivity of combined measurement and modeling process. Adding an extra interfacial layer between the cap GaAs and the AlAs layers did improve the fits somewhat, but in a nonphysical way. The interfacial layer tended to dominate, and the cap thickness solved to zero thickness. The resultant models implied an Al presence of $10 \%$ or more throughout the cap which is very difficult to justify for MBE grown samples. The principal effect of the Al presence in the model is to shift the $\mathrm{CP}$ structure of the cap optical constants to higher energies. However, this shift can also be explained without requiring the presence of $\mathrm{Al}$ by assuming that the dielectric function for these very thin layers may be influenced by a quantum thickness effect. For the remainder of this work, all the models assumed perfect interfaces. Any real interfacial effects are therefore included in the fitted optical constants.

As an intermediate step, the GaAs cap was modeled using a group of six oscillators as in Eq. (4). This allowed the cap optical constants to be adjusted using many fewer parameters than if wavelength tabulated values were used. Initially, a good fit was found to bulk GaAs in the region from 1.8 to $5.0 \mathrm{eV}$. Below $1.8 \mathrm{eV}$ the oscillators could not model the $E_{0} \mathrm{CP}$ structure. To keep the various fits easily comparable, the same data set was used in each case, hence the limited range described previously. Fit 2 was the same as 1 except that the oscillator model for bulk GaAs was used for the caps. The resulting AlAs optical constants (not shown) were little changed except in the UV, where the oscillator fit to the bulk GaAs was less accurate. The $\chi^{2}$ and layer thicknesses were essentially unchanged (Table II), indicating the applicability of the oscillator cap as a starting point.

In fit 3 , the three parameters (amplitude, broadening, and energy) for both the $E_{1}$ and $E_{1}+\Delta_{1}$ oscillators were allowed 


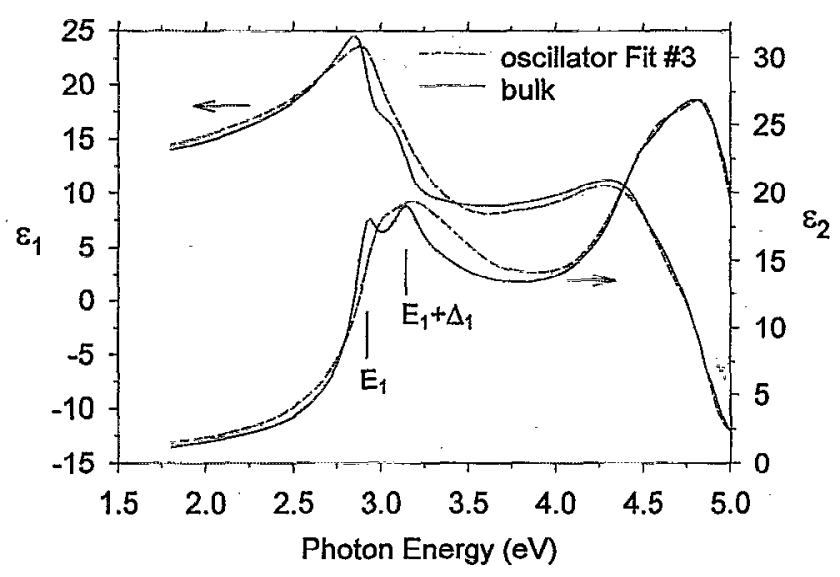

FIG. 6. Comparison of bulk GaAs optical constants used for the substrate and the cap GaAs optical constants used for fit 3. The cap optical constants are modeled using an ensemble of oscillators. Only the oscillator parameters describing the $E_{1}$ and $E_{1}+\Delta_{1}$ critical points were fit. The results indicate a blue shift and broadening of these oscillators.

to vary. By fitting just these six additional parameters, which affect the cap optical constants around $3 \mathrm{eV}, \chi^{2}$ was reduced another order of magnitude and the ex situ fits were nearly perfect over the entire range. For the GaAs caps, the bulklike
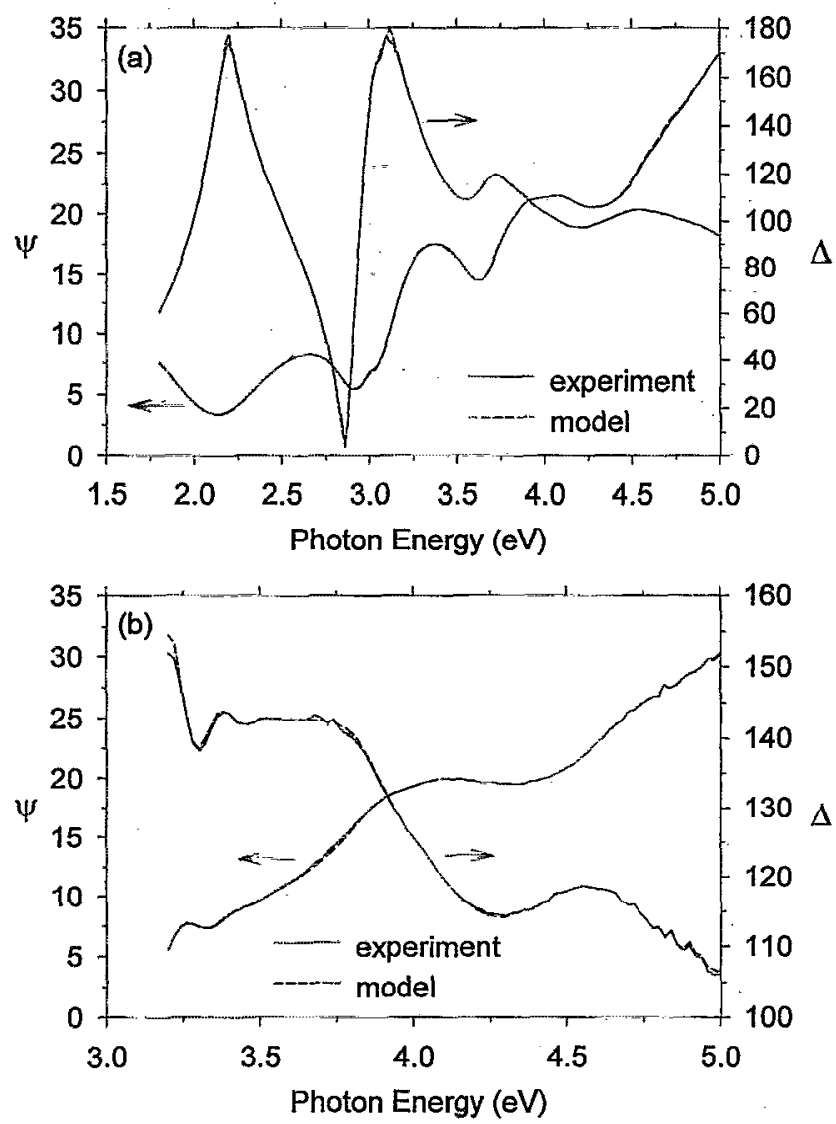

FIG. 7. The experimental data and best model fits (a) for ex situ sample 7357 and (b) for the in situ sample are shown for fit 4. The optical constants of both the AlAs and cap GaAs were fit at each wavelength. The ex situ oxide and cap thicknesses (not fit) were fixed at the results from fit 3 . The fits for 7358 and 7179 (not shown) are of the same quality. and fit 3 oscillator parameters are given in Table $\mathrm{II}$, and a comparison of optical constants is shown in Fig 6. The parameter changes indicate a blue shift in energy and increased broadening. The blue shift is qualitatively consistent with published $E_{1}$ and $E_{1}+\Delta_{1}$ shifts in GaAs quantum wells (QWs). ${ }^{29,30}$ The question is, can a thin GaAs cap be considered similar to a QW? Clearly the bottom half of a cap layer looks like a QW with AlAs barriers. Although very different, the top half also exhibits carrier confinement via the oxide and vacuum levels. The increased broadening may be due to the presence of the oxide-cap interface reducing lifetimes of states involved with the $\mathrm{CP}$ transitions. This modification of optical constants for thin caps may have implications for in situ growth control techniques which use the $E_{1}$ and $E_{1}+\Delta_{1}$ critical-point region to determine composition. ${ }^{31} \mathrm{~A}$ problem might exist since the surface layer optical constants would be a function of thickness for a period of time after the change from wide-gap to narrow-gap material at a heterointerface. The corresponding fit 3 AlAs values become closer in amplitude to the published values above the gap and stay very close to the prism values below the gap. Additionally, the anomalous feature at 3.7 is greatly reduced.

The cap oscillator fit was used as an intermediate step to demonstrate the difference between thin cap GaAs and bulk GaAs optical constants, and to determine appropriate cap and oxide layer thicknesses. For fit 4 , the cap and oxide thicknesses were fixed at their fit 3 values in anticipation of strong correlation between cap optical constants and cap/oxide thicknesses. Thus for fit 4 , two sets of optical constants (GaAs cap and AlAs) were fit at all wavelengths in addition to five of the structural parameters. The $\chi^{2}$ was reduced by a factor of 4 while the AlAs thicknesses were almost unchanged. The essentially perfect fit quality is shown for two of the samples in Fig. 7. To produce the tinal AlAs values of fit 5 , the structural parameters from fit 4 were used as constants, the full data sets from 1.42 to $5.0 \mathrm{eV}$ were used, and both the AlAs and GaAs cap optical constants were fit on a wavelength-by-wavelength basis. (With thicknesses fixed, the data at each wavelength can be fit independently. Only

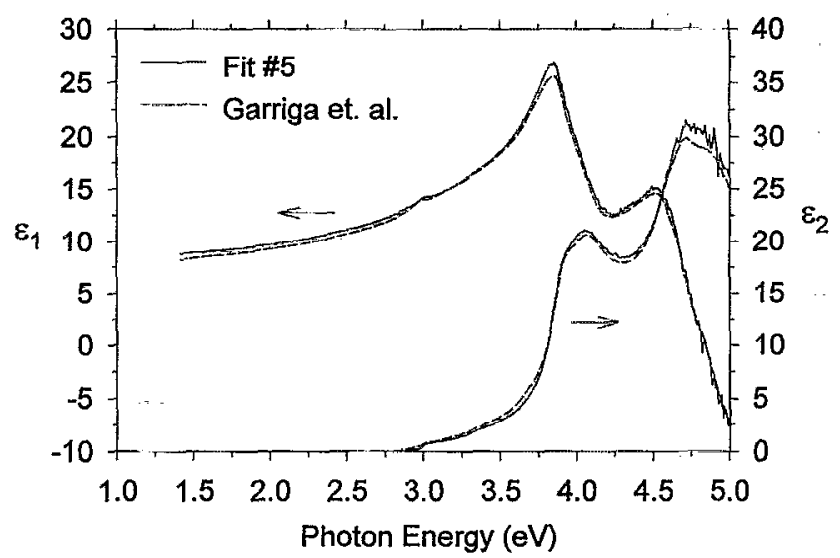

FIG. 8. The AlAs optical constants from fit 5 are compared with previously published values also determined by spectroscopic ellipsometry (Ref. 6). These results should be compared with Fig. 3. The oftset in $\epsilon_{1}$ below $3 \mathrm{eV}$ remains. The difference in $\epsilon_{2}$ above $3.8 \mathrm{eV}$ is smaller. The anomalous feature at $3.7 \mathrm{eV}$ seen in the fit 1 spectra is no longer present. 


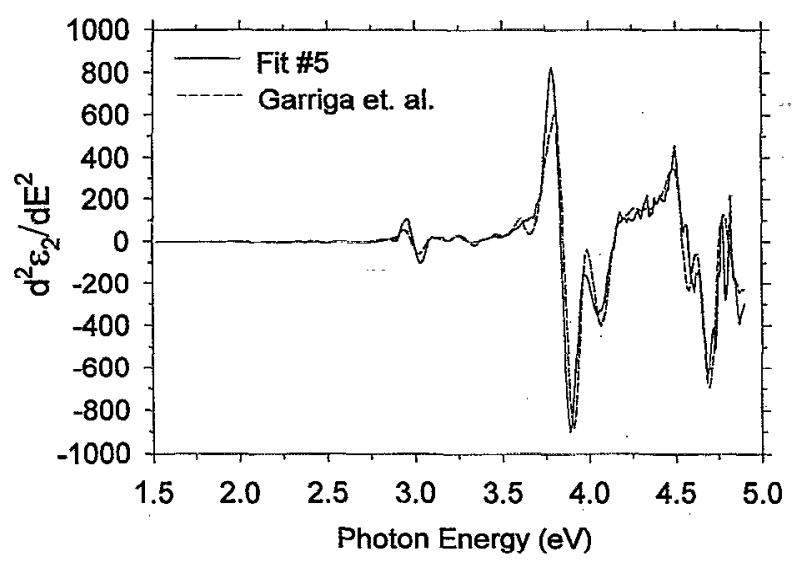

FIG. 9. The second derivative of the imaginary part of the AlAs dielectric function from fit 5 are compared with similarly treated published values. The derivative spectra is similar in both shape and amplitude. The difference in $\epsilon_{2}$ above $3.8 \mathrm{eV}$ (Fig. 7) is just an offset due to differences in modeling overlayers in ellipsometric data and is not due to a difference in criticalpoint structure.

the four parameters at each wavelength needed to be fit simultaneously.) The final AlAs values match the published values closely in absorption amplitude and second derivative as seen in Figs. 8 and 9, respectively. The anomalous derivative feature at $3.7 \mathrm{eV}$ seen in fit 1 is no longer present. The only major difference is in the below-gap dielectric constant where our values from 1.42 to $2.2 \mathrm{eV}$ closely match the published prism measurements. The relatively large noise in our data above $4.5 \mathrm{eV}$ is directly related to the noise from the in situ measurements which is fit almost exactly as seen in Fig. 7. The ex situ samples are relatively insensitive to this noise and they contribute primarily to the general amplitude of $\epsilon_{2}$ in this spectral region. This is an example where a good parametric model for semiconductor optical constants, if developed, might be employed to extract smooth and physically meaningful results from noisy data. The concurrently extracted GaAs cap optical constants exhibit differences from bulk values as seen in Fig. 10. Some small anomalous structure is apparent in the $3.5-4.0 \mathrm{eV}$ region. However,

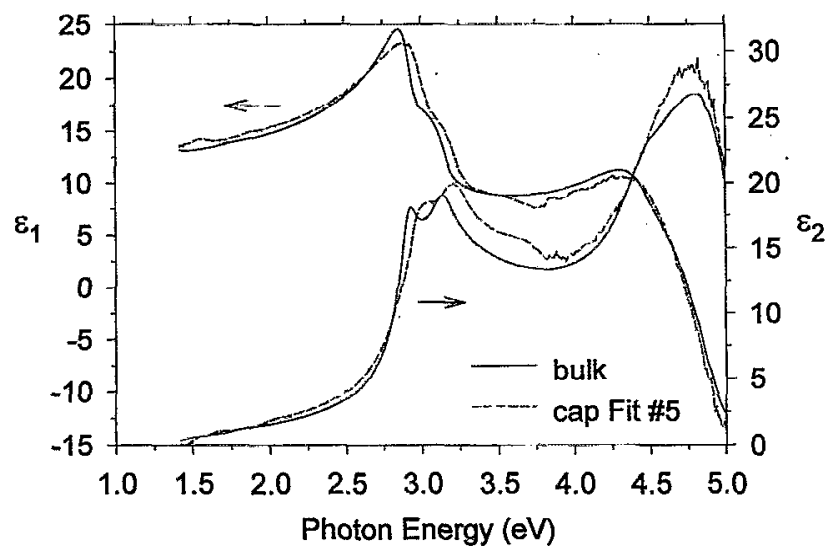

FIG. 10. Comparison of bulk GaAs optical constants used for the substrate and the cap $\mathrm{GaAs}$ optical constants determined from fit 5 . The results indicate a blue shift and broadening of the $E_{1}{ }^{-}$and $E_{1}+\Delta_{1}$ critical points.

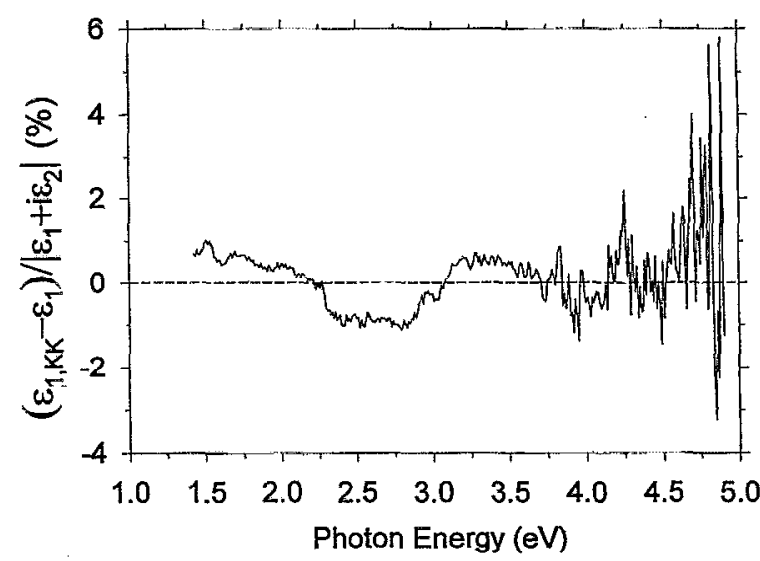

FIG. 11. The difference between the measured $\epsilon_{1}$ values and a KramersKronig model using the fit 5 AlAs values is shown. The model was fit to the $\epsilon_{1}$ values from 1.42 to $4.5 \mathrm{eV}$ with a resulting error of about $1 \%$ or less in this region. There is a noticeable (larger than noise) systematic error in this region.

recall that these values relied upon only three layers, each less than $50 \AA$ thick, so that sensitivity is low relative to the AlAs layer optical constants. Under these conditions, some small anomalies in the GaAs cap optical constants are to be expected, even for a very good model. The amplitude differences in the UV $(4.5-5.0 \mathrm{eV})$ can be attributed to features not accounted for in the model such as microstructure roughness or interface grading. However, such model imperfections cannot explain the energy blue shift of the $E_{1}$ and $E_{1}+\Delta_{1}$ structure, which we attribute to a thickness quantization effect at the surface. The $E_{1}$ and $E_{1}+\Delta_{1}$ CPs also appear slightly broadened when compared to bulk values, but are somewhat sharper when compared to the oscillator model in Fig. 6. However, we do not feel justified in making quantitative claims on this apparent broadening increase.

The final AIAs optical constants shown in Fig. 8 were subjected to a $\mathrm{K}-\mathrm{K}$ consistency check using the following model:

$$
\begin{aligned}
\epsilon_{\mathrm{l}}^{\mathrm{KK}}(\hbar \omega)= & \epsilon_{\mathrm{l}}^{\mathrm{offset}}-\frac{A_{0}}{(\hbar \omega)^{2}-E_{0}^{2}} \\
& +\frac{2}{\pi} P \int_{1.4 \mathrm{eV}}^{5.0 \mathrm{eV}} \frac{x \epsilon_{2}^{\text {meas }}(x)}{x^{2}-(\hbar \omega)^{2}} d x .
\end{aligned}
$$

The zero width oscillator with amplitude $A_{0}$ was added to account for absorption above, but near, $5 \mathrm{eV}$ while $\epsilon_{1}^{\text {offset }}$ accounts for absorption far above $5 \mathrm{eV}$. Because the $\epsilon_{2}$ spectrum extends only to $5.0 \mathrm{eV}$, the $\mathrm{K}-\mathrm{K}$ model cannot be very accurate near this boundary. The three parameters, $A_{0}(47.36$ $\left.\mathrm{eV}^{2}\right), E_{0}(5.235 \mathrm{eV})$, and $\epsilon_{1}^{\text {offset }}(2.1492)$, were fit to $\epsilon_{1}$ values from 1.42 to $4.5 \mathrm{eV}$ of fit 5 . The $\mathrm{K}-\mathrm{K}$ modeled values are compared with the experimental values in Fig. 11 from 1.42 to $5.0 \mathrm{eV}$, demonstrating a self-consistency of $1 \%$ in $\left|\epsilon_{1}+i \epsilon_{2}\right|$ over most of the spectral region. Above $4.5 \mathrm{eV}$, the $\epsilon_{2}$ data were noisy and the $\epsilon_{1}$ values were not used in fitting the $\mathrm{K}-\mathrm{K}$ model parameters. The $\mathrm{K}-\mathrm{K}$ model was also used to extend $\epsilon_{1}$ down to $0.6 \mathrm{eV}$, and these values compare well with prism measurements as seen in Fig. 12. 


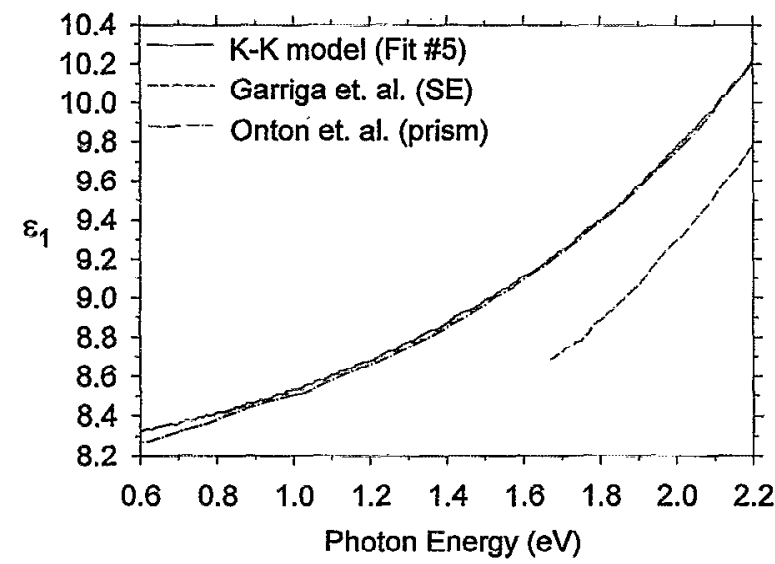

FIG. 12. The Kramers-Kronig (K-K) model (see Fig. 11) for fit 5 is compared with previously published ellipsometric (Ref. 6) and prism measurements (Ref. 12). The $\mathrm{K}-\mathrm{K}$ model extended below the measurement range is in agreement $\left(<0.1\right.$ in $\left.\epsilon_{1}\right)$ with the prism measured values down to $0.6 \mathrm{eV}$.

\section{EXTENDED ANALYSIS}

To demonstrate the correlation of cap optical constants and thicknesses, fit 6 was performed in the same manner as fit 4 except that all thicknesses were fit in addition to the AlAs and cap GaAs optical constants. The $\chi^{2}$ dropped only fractionally and the AlAs values were essentially unchanged, but the cap thicknesses (Table II) and optical constants (not shown) were noticeably different. This demonstrates that the overlayer thicknesses are not uniquely determined unless an additional assumption about the GaAs cap optical constants is made as was done in fit 3 . In that case we forced the cap to be bulklike except in the vicinity of $E_{1}$. But fit 6 also shows that the multisample analysis can produce the same AlAs values regardless of the precise overlayer model. The AlAs layers did not show the same correlation problems as the cap because the interference oscillations in the transparent region had to be fit with the same optical constants for all three samples. The cap and oxide layers are too thin to produce their own internal interference features. The inclusion of the in situ sample data left the UV AlAs optical constants unchanged from fits 4 to 6 .

Fit 7 was performed to demonstrate that our fit $4 \mathrm{AlAs}$ optical constants are truly different from previously published ellipsometric values; the differences are not just a result of our modified GaAs cap optical constants. For this fit, previously published AlAs values were used and the GaAs cap optical constants were fit. If the quality of this fit approached that of fit 4 , then our fit 4 optical constants and the Garriga values would not be distinguishable using these samples. However, the fit was much worse, and, moreover, the resulting thicknesses are very much out of line because the fitted cap optical constants (not shown) were totally unphysical. The difference in the below-gap $\epsilon_{1}$ values, shown in Figs. 8 and 12, is similar in magnitude to the differences in $\epsilon_{2}$ above $4.5 \mathrm{eV}$. However, the below-gap differences are critical when attempting to fit the ex situ data because of the interference patterns created. The $\epsilon_{2}$ difference above $4.5 \mathrm{eV}$ occurs due to different assumptions about the overlayer structure. Using both the previous AlAs values (fit 0 ) and our
TABLE IV. Structural parameter fit results when fitting each sample separately.

\begin{tabular}{clcc}
\hline \multirow{2}{*}{ Sample } & Cap & Not fit & Fit \\
& $M$ & 3 & 9 \\
\hline \multirow{7}{*}{7358} & $\xi^{2}$ & 1590.0 & 216.0 \\
& $t$-ox & $31.1(0.18)$ & $31.5(0.10)$ \\
& $t$-cap & $43.0(0.23)$ & $43.6(0.42)$ \\
& $t$-AlAs & $716.1(0.46)$ & $708.4(0.50)$ \\
7357 & $\xi^{2}$ & 599.0 & 110.0 \\
& $t$-ox & $32.8(0.17)$ & $33.3(0.12)$ \\
& $t$-cap & $40.7(0.23)$ & $41.4(0.46)$ \\
& $t$-AlAs & $818.8(0.45)$ & $820.4(0.53)$ \\
7179 & $\xi^{2}$ & 787.0 & 90.0 \\
& $t$-ox & $30.1(0.18)$ & $32.1(0.12)$ \\
& $t$-cap & $50.1(0.22)$ & $46.6(0.32)$ \\
& $t$-AlAs & $994.0(0.50)$ & $1000.5(0.33)$ \\
& $\xi^{2}$ & 809.0 & \\
7180 & $t$-ox & $30.8(0.12)$ & $31.6(0.11)$ \\
& $t$-cap & $47.3(0.22)$ & $45.4(0.41)$ \\
& $t$-AlAs & $959.1(0.51)$ & $966.0(0.50)$ \\
\hline \hline
\end{tabular}

values (fit 5) one can fit the in situ data and, therefore, the absolute magnitude in $\epsilon_{2}$ is not completely determined. Ellipsometry alone has difficulty precisely measuring $\epsilon_{2}$ peak amplitudes when working with samples with overlayers. Additional information or assumptions are need for a unique determination. For fitting ellipsometric data and determining layer thicknesses, we prefer our fit 5 values because of their demonstrated ability to fit more than one kind of sample containing AlAs. The below-gap dielectric values are very important for determining accurate AlAs layer thicknesses. For accurate determination of $\mathrm{CP}$ energies, the Garriga data are preferred since lower noise UV ellipsometric data were obtained for their in situ sample. The precise overlayer assumptions and below-gap index values used to model the Garriga data have little effect on a $\mathrm{CP}$ analysis.

Fit 8 examined the necessity of using nonbulk GaAs cap optical constants if only thickness determinations were desired. In most applications of ellipsometry, one is trying to evaluate thicknesses using already available optical constants. For this fit, bulk GaAs was used for the substrate and for the cap and fit $5 \mathrm{AlAs}$ values were used. Only the 13 structural parameters were fit. The resulting thicknesses were somewhat closer to the fit 4 values than were the base line (fit 0) values; however, the fit quality was poor as the $\chi^{2}$ value in Table II indicates. Thus, to fully describe the $e x$ situ samples with thin GaAs caps, the cap optical constants must be allowed to vary. This highlights the utility of the oscillator modeled cap, where only six extra parameters are needed to achieve a good fit (fit 3 ) and therefore believable thicknesses.

To further examine the role of fitting the cap optical constants, each of the ex situ samples, including 7180 $\left(450{ }^{\circ} \mathrm{C}\right.$ growth temperature), were fit individually using models as in fits 2 and 3. The cap was modeled as an ensemble of oscillators and the AlAs optical constants were taken from fit 5. The results of the fits are given in Table IV for two cases: column 3, fitting the thicknesses only (like fit 2 ), and column 4, fitting the thicknesses and six parameters 


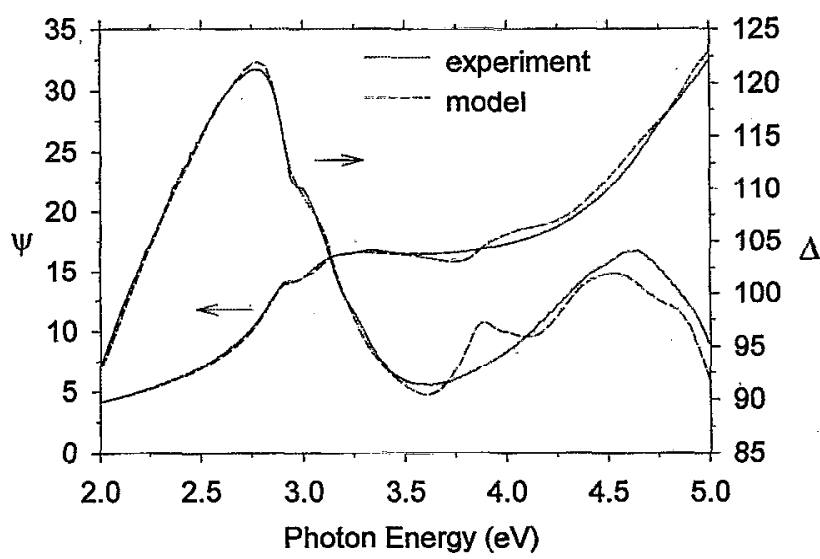

FIG. 13. Shown are experimental and fit model values for a sample containing a nominally $20 \AA$ thick AlAs in a background of GaAs. Near $3.9 \mathrm{eV}$ the $E_{1}$ and $E_{1}+\Delta_{1}$ critical-point structure is clearly present in the model but is completely smeared out in the experimental data. The bulk AlAs optical constants determined from fit 5 are not a good representation for a thin $(\sim 20$ $\AA$ ) isolated AlAs barrier.

for the $E_{1}$ and $E_{1}+\Delta_{1}$ oscillators (like fit 3). The resulting thicknesses are not strongly dependent on the fitting technique, but $\xi^{2}$ is much lower when fitting the cap oscillators. The $\xi^{2}$ values are generally higher for these fits than for fits 2 and 3 , but this can be attributed to the fact that the AlAs optical constant being used came from fit 5 where the GaAs cap optical constants were also fit at each wavelength. The quality of the fits to 7180 are similar to those for the other ex situ samples, and we therefore judge the optical constants from fit 5 applicable to AlAs grown from 450 to $600{ }^{\circ} \mathrm{C}$.

\section{APPLICATION TO VERY THIN AIAS LAYERS}

The optical constants from fit 5 have been shown to be valid for layer thicknesses greater than $700 \AA$. However, we have noted difficulty in fitting a sample with a nominally 20 $\AA$ AlAs layer. This sample (7223) had a nominal structure of $20 \AA$ of AlAs on a GaAs buffer with a GaAs cap of $20 \AA$. The fit model used was the same as for the ex situ samples in Fig. 1 and used an oscillator ensemble for the cap optical constants. The nominal $20 \AA$ AlAs thickness was used, but the cap and oxide thicknesses were fit, as were the broadening and energy parameters of the cap $E_{1}$ and $E_{1}+\Delta_{1}$ oscillators. The resulting fit (Fig. 13) was reasonable in the GaAs $E_{1}$ and $E_{1}+\Delta_{1}$ region $(3 \mathrm{cV})$ but was obviously poor around the $E_{1}$ and $E_{1}+\Delta_{1}(3.9 \mathrm{eV})$ CPs for AlAs. The AlAs criticalpoint structure, near $3.9 \mathrm{eV}$, is clearly present in the model but not in the data.

The AlAs optical constants are apparently not bulklike because it seems unreasonable to believe modifying the optical constants of the GaAs cap could cancel out the AlAs structure. Also, the data could not be fit by assuming the AlAs was inadvertently left out of the growth sequence; there is indeed an extra layer present. The $\Lambda \mathrm{I} A \mathrm{~s}$ was an isolated barrier and this smearing out of the $E_{1}$ and $E_{1}+\Delta_{1}$ critical-point structure may be due to a quantum-mechanical thin layer effect. The states involved in the $E_{1}$ and $E_{1}+\Delta_{1}$ structure could leak into the surrounding GaAs, reducing the state lifetimes and broadening the transition. Since this criti- cal point structure is thought to involve saddle-point excitons, ${ }^{32}$ this might explain why these two critical points are so strongly affected. A systematic study of barrier thicknesses from a few tens of angstroms to a few hundred angstroms might clarify the effect. If smearing due to thinness is indeed the case, this would be generally applicable to other material systems as well. (We have noted similar effects for thin strained AlAs and AlSb layers. ${ }^{33}$ ) This effect is the converse of that seen in GaAs QWs where the $E_{1}$ and $E_{1}+\Delta_{1}$ states are confined by the surrounding material causing an energy shift, but little broadening. ${ }^{29,30}$ This effect, like the thin GaAs cap effect, could be an important consideration for in situ growth control applications which make use of optical constants at these critical points.

\section{CONCLUSIONS}

Using variable angle spectroscopic ellipsometry, we have determined optical constants for AlAs $(1.4-5.0 \mathrm{eV})$ that are simultaneously compatible with measured data from three GaAs-capped and one As-decapped sample. The second derivative spectra are compatible with published values at and above the direct gap at $3 \mathrm{eV}$. Our below-gap index values are in good agreement with published prism measured values. The AlAs spectrum is Kramers-Kronig self-consistent over the measured range and is compatible with published values from 0.6 to $1.4 \mathrm{eV}$. We have demonstrated that optical constants for thin $(<50 \AA)$ GaAs caps on AlAs are sufficiently different from bulk GaAs values as to require special consideration in the model fits. For the thin GaAs caps, the $E_{1}$ and $E_{1}+\Delta_{1}$ critical-point structure is shifted to higher energies. This is indicative of a quantization effect at the surface, and is qualitatively similar to published observations for GaAs quantum wells. We have also shown that bulk AlAs optical constants do not adequately represent thin $(\sim 20$ $\AA)$ AlAs barrier layers in GaAs. This barrier broadening effect and the thin cap effects have implications for in situ growth control applications where the surface layer optical constants could be a function of thickness when growing heterointerfaces, especially in the $E_{1}$ and $E_{1}+\Delta_{1}$ criticalpoint region.

\section{ACKNOWLEDGMENTS}

We wish to thank Dr. Manuel Garriga for providing us his AlAs optical constants in digital form. This work was supported by ARPA consortium agreement No. MDA972-93H-005.

${ }^{1}$ D. E. Aspnes, S. M. Kelso, R. A. Logan, and R. Bhat, J. Appl. Phys. 60 , 754 (1986).

${ }^{2}$ G. E. Jellison, Jr., Opt. Mater. 1, 41 (1992).

${ }^{3}$ G. E. Jellison, Jr., Opt. Mater. 1, 151 (1992).

${ }^{4}$ C. M. Herzinger, P. G. Snyder, B. Johs, and J. A. Woollam, J. Appl. Phys. 77, 1715 (1995)

${ }^{5}$ M. Garriga, P. Lautenschlager, M. Cardona, and K. Ploog, Solid State Commun. 61, 157 (1987).

${ }^{6}$ M. Garriga, M. Kelly, and K. Ploog, Thin Solid Films 233, 123 (1993).

${ }^{7}$ H. Yao, P. G. Snyder, K. Stair, and T. Bird, Mater. Res. Soc. Symp. Proc. 242, 481 (1992).

${ }^{8} \mathrm{M}$. Malin and K. Vedam, in Proceedings of the Third International Conference on Ellipsometry, University of Nebraska, September 1975, edited 
by N. M. Bashara and R. M. A Azzam (North-Holland, Amsterdam, 1975), p. 49.

${ }^{9} \mathrm{~W} . \mathrm{G}$. Oldham, in Proceedings of the Symposium on recent Developments in Ellipsometry, University of Nebraska, August 1968, edited by N. M. Bashara, A. B. Buckman, and A. C. Hall (North-Holland, Amsterdam, 1968), p. 97.

${ }^{10}$ W. A. McGahan, B. Johs, and J. A. Woollam, Thin Solid Films 234, 443 (1993).

"N. V. Nguyen, D. Chandler-Horowitz, P. M. Amirtharaj, and J. G. Pelliegrino, Appl. Phys. Lett. 64, 2688 (1994).

${ }^{12}$ R. E. Fern and A. Onton, J. Appl. Phys. 42, 3499 (1971).

${ }^{13}$ R. M. A. Azzam and N. M. Bashara, in Ellipsometry and Polarized Light (North-Holland, Amsterdam, 1977), Chap. 4.

${ }^{14}$ W. H. Press, B. P. Flannery, S. A. Teukolsky, and W. T. Vetterling, in Numerical Recipes: The Art of Scientific Computing (Cambridge University, Cambridge, 1988), Chap. 14.

${ }^{15}$ G. E. Jellison, Jr., Appl. Opt. 30, 3354 (1991).

${ }^{16}$ B. Johs, W. A. McGahan, and J. A. Woollam, in Proceedings of the 1994 ICMCTF, San Diego, April 1994 (in press).

${ }^{17}$ S. Zollner, Appl. Phys. Lett. 63, 2523 (1993).

${ }^{18}$ C. C. Kim, J. W. Garland, and P. M Raccah, Phys. Rev. B 47, 1876 (1993)

${ }^{19} \mathrm{~F}$. Wooten, Optical Properties of Solids (Academic, New York, 1972).

${ }^{20} \mathrm{M}$. Erman, J. B. Theeten, P. Chambon, S. M. Kelso, and D. E. Aspnes, J. Appl. Phys. 56, 2664 (1984).

${ }^{21}$ H. D. Yao, P. G. Snyder, and J. A. Woollam, J. Appl. Phys. 70, 3261 (1991).

${ }^{22}$ F. Terry, Jr., J. Appl. Phys. 70, 409 (1991).
${ }^{23}$ P. G. Snyder, M. C. Rost, G. H. Bu-Abbud, and J. A. Woollam, J. Appl. Phys. 60, 3293 (1986).

${ }^{24}$ W. A. McGahan, T. Makovicka, J. Hale, and J. A. Woollam, Surf. Coat. Tech. 62, 707 (1993).

${ }^{25}$ The GaAs dielectric function used in this work came from ellipsometric measurements of a (100) substrate with native oxide using the approach presented in Ref. 4. The oxide thickness was determined using published absorption measurements at energies just above the direct band gap from D. S. Gerber and G. N. Maracas, IEEE J. Quantum Electron. 29, 2589 (1993).

${ }^{26}$ G. N. Greaves, E. A. Davis, and J. Bordas, Philos. Mag. 34, 265 (1976).

${ }^{27}$ D. E. Aspnes, in Handbook of Optical Constants of Solids, edited by E. D. Palik (Academic, Orlando, 1985), Chap. 5, p. 104.

${ }^{28}$ F. A. Jenkins and H. E. White, Fundamentals of Optics (McGraw-Hill, New York, 1957).

${ }^{29}$ M. Erman, J. B. Theeten, P. Frijlink, S. Gaillard, F. J. Hia, and C. Alibert, J. Appl. Phys. 56, 3241 (1984).

${ }^{30}$ R. P. Vasquez, R. T. Kuroda, and A. Madhukar, J. Appl. Phys. 61, 2973 (1987).

${ }^{31}$ D. E. Aspnes, W. E. Quinn, M. C. Tamargo, M. A. A. Pudensi, S. A. Schwarz, M. J. S. P. Brasil, R. E. Nahory, and S. Gregory, Appl. Phys. Lett. 60, 1244 (1992).

${ }^{32}$ J. C. Phillips, Solid State Phys. 18, 128 (1966); M. Chandraesekhar and F. H. Pollak, Phys. Rev. B 15, 2127 (1977).

${ }^{33}$ C. M. Herzinger, P. G. Snyder, F. G. Celii, Y.-C. Kao, D. Chow, B. Johs, and J. A. Woollam (unpublished). 